\title{
GLOBAL PROPERTIES OF ROTATING NEUTRON STARS WITH QCD EQUATIONS OF STATE
}

\section{Gorda, Tyler}

2016-11-20

Gorda , T 2016 , ' GLOBAL PROPERTIES OF ROTATING NEUTRON STARS WITH QCD

EQUATIONS OF STATE ' , Astrophysical Journal , vol. 832 , no. 1 , 28 . https://doi.org/10.3847/0004-637X/832/1/28

http://hdl.handle.net/10138/232686

https://doi.org/10.3847/0004-637X/832/1/28

unspecified

publishedVersion

Downloaded from Helda, University of Helsinki institutional repository.

This is an electronic reprint of the original article.

This reprint may differ from the original in pagination and typographic detail.

Please cite the original version. 


\title{
GLOBAL PROPERTIES OF ROTATING NEUTRON STARS WITH QCD EQUATIONS OF STATE
}

\author{
Tyler GORDA ${ }^{1,2}$ \\ ${ }^{1}$ University of Colorado Boulder, Boulder, Colorado, USA; tyler.gorda@ helsinki.fi \\ ${ }^{2}$ University of Helsinki, Helsinki, Finland \\ Received 2016 July 6; revised 2016 September 9; accepted 2016 September 9; published 2016 November 14
}

\begin{abstract}
We numerically investigate global properties of rotating neutron stars (NSs) using the allowed band of QCD equations of state derived by Kurkela et al. This band is constrained by chiral effective theory at low densities and perturbative QCD at high densities, and is thus, in essence, a controlled constraint from first-principles physics. Previously, this band of equations of state was used to investigate non-rotating NSs only; in this work, we extend these results to any rotation frequency below the mass-shedding limit. We investigate mass-radius curves, allowed mass-frequency regions, radius-frequency curves for a typical $1.4 M_{\odot}$ star, and the values of the moment of inertia of the double pulsar PSR J0737-3039A, a pulsar for which the moment of inertia may be constrained observationally in a few years. We present limits on observational data coming from these constraints, and identify values of observationally relevant parameters that would further constrain the allowed region for the QCD equation of state. We also discuss how much this region would be constrained by a measurement of the moment of inertia of the double pulsar PSR J0737-3039A.
\end{abstract}

Key words: dense matter - equation of state - stars: neutron

\section{INTRODUCTION}

Neutron stars (NSs) are one of the most extreme physical systems in the cosmos. Within a sphere of radius $\sim 10 \mathrm{~km}$, there lies over $1 M_{\odot}$ of matter. In the outer layers of NSs, controlled techniques such as chiral effective theory (ChEFT; Tews et al. 2013) or quantum Monte Carlo (Abbar et al. 2015) are applicable and can yield insights into both the static properties of the bulk matter (such as the equation of state or EoS) and some transport properties. Currently, these low-density calculations are valid up to about 1.1 times the nuclear saturation density $n_{s} \approx 0.16 / \mathrm{fm}^{3}$, corresponding to a baryon chemical potential of about $\mu_{B} \approx 0.97 \mathrm{GeV}$ (Tews et al. 2013). Deep in the core, however, such controlled, direct theoretical calculations are not possible. This is because the densities and chemical potentials at the center of the star, though extreme, are not large enough to fall into the range accessible by perturbative quantum chromodynamics (pQCD). In the stateof-the-art pQCD calculations at zero temperature in Kurkela et al. (2010a), the errors associated with varying the mass scale reach $30 \%$ at around $\mu_{B}=2.6 \mathrm{GeV}$. The value of $\mu_{B}$ in the cores of NSs lie within a subset of this $0.97-2.6 \mathrm{GeV}$ range.

The problem of the interiors of NSs is thus currently a nonperturbative one. However, one can hope to reach the intermediate values of $\mu_{B}$ by matching the low-density EoS from the low-energy effective theories to the pQCD results in a thermodynamically consistent way to investigate the (static) makeup of NSs. This has been carried out in the work of Kurkela et al. (2014) and Fraga et al. (2016), who, in addition, incorporated the $2 M_{\odot}$ constraint from Demorest et al. (2010), and Antoniadis et al. (2013; see also Hebeler \& Schwenk 2010, in which the authors use only ChEFT and the $2 M_{\odot}$ constraint to extend the low-energy EoS). In these works, the authors used their matched EoSs to analyze non-rotating NSs only. It is known (Benhar et al. 2005; Cipolletta et al. 2015) that slowly rotating NSs can be approximated as non-rotating for frequencies of rotation less than about $f \approx 200 \mathrm{~Hz}$. Beyond this, however, one must use numerical codes to analyze the structure of the stars. Such a numerical approach has recently been used by Cipolletta et al. (2015) and Haensel et al. (2016) in the context of phenomenological EoSs. One of the main purposes of the current article is to extend these analyses to include EoSs that are more fully constrained by firstprinciple physics. Broadly speaking, the purpose of this work is to investigate the effects of rotation on NSs all the way up to the mass-shedding limit using the constraints on the QCD EoS determined in Kurkela et al. (2014) and Fraga et al. (2016). We are particularly interested in constraining NS properties that are relevant observationally. As such, we investigate the maximum allowed NS masses, and the allowed regions for mass-radius curves, mass-frequency curves, and radius-frequency curves for a typical $1.4 M_{\odot}$ star. In addition, we investigate the allowed values of the moment of inertia of the double pulsar PSR J0737-3039A (Morrison et al. 2004; Kramer \& Wex 2009) and study how this is correlated with the radius. In this way, we hope that this work will provide the literature with strong direct links between astronomical observations and the allowed QCD EoSs coming from current state-of-the-art pQCD and ChEFT calculations.

We note at the outset that even though the pQCD result of Kurkela et al. (2010a) assumed local charge neutrality, this does not actually imply that the EoS band of Kurkela et al. (2014) makes this assumption. There are two reasons for this. First, as was noted in Kurkela et al. (2010a), switching between local and global charge neutrality for the pQCD EoS typically leads to a smaller variation in the pressure than is already included in the renormalization scale dependence (Glendenning 2000). Second, since the polytropic matching carried out in Kurkela et al. (2014) does not preclude the formation of a mixed phase at the matching points (see, e.g., Glendenning 1992), there is in principle no assumption of local charge neutrality made for the band of EoSs that we use in this work.

The structure of this paper is as follows. In Section 2, we briefly review the RNS code and describe how it was used to construct all of the aforementioned relations between the NS properties listed above. In Section 3, we present our results and all of our plots. In our concluding Section 4, we review our 
main findings, including ones that are most relevant to astrophysical observation.

\section{METHODOLOGY}

To conduct our analysis, we used the publicly available RNS code. It can take, as input, an EoS in the form of $P(\varepsilon)$ and two parameters: a central energy density $\varepsilon$ and the ratio of the polar coordinate radius to the equatorial coordinate radius $r$. Other inputs can be used as well (see below), but internally each NS that is constructed and specified by the parameters $\varepsilon$ and $r$. From this input, the code can calculate various global properties of the star, including the total (or gravitational) mass $M$, the circumferential equatorial radius $R_{\mathrm{e}}$, the frequency of rotation $f$, and the moment of inertia $I$.

In addition to constructing a single star specified by $\varepsilon$ and $r$, the RNS code can construct sequences of stars as well as accept other stellar properties as input to construct internal sequences and find stars satisfying those inputs. It can also calculate the mass-shedding frequency for a given central energy density $\varepsilon_{0}$, which is the fastest rotation rate possible before the star begins to throw off mass from its equator. This provides an upper bound on the rotation rate for the central energy density $\varepsilon_{0}$. Rotating stars have both a larger maximum mass and a larger maximum equatorial radius, and so the mass-shedding limit can be used to investigate larger, more massive stars than were possible in the non-rotating limit.

The approach used in this investigation was to take the EoSs used in Kurkela et al. (2014) and Fraga et al. (2016) in the form $P(\varepsilon)$ and feed them into the RNS code to calculate various properties of physical interest. A comment is in order here. In Kurkela et al. (2014) and Fraga et al. (2016), the authors match the ChEFT EoSs to the pQCD band using two or three intermediate polytropic EoSs. In addition, they perform the matching both with and without latent heat at the matching points of the polytropic EoSs. In the end, the authors conclude that adding latent heat is actually more restrictive on the matching, and, in addition, they found that a third polytrope only minimally increased the range of allowed EoSs. In light of these results, we have also only used the bitropic EoSs without latent heat in this work.

To construct our data, we first ran the RNS code on the static and mass-shedding sequences. From this, we could construct the mass-radius curves and one boundary of the allowed massfrequency region for NSs. The rest of our numerical data involved either fixed-frequency runs, fixed-mass runs (or both), or coding a binary search to fill in the gaps where the code was unable to generate the star. This was necessary in the cases of very small frequencies because, internally, the code always uses $r$ as a parameter instead of $f$. (This behavior was also noted in Cipolletta et al. 2015.) The fixed-frequency runs were used to determine the other boundary of the allowed mass-frequency region, and the fixed-mass runs were used to determine the radius-frequency relations for a typical, $1.4 M_{\odot}$ NS. Finally, the fixed-mass-and-frequency runs were used for investigating PSR J0737-3039A.

\section{RESULTS}

We first present our results for mass versus equatorial radius curves in Figure 1. The non-rotating region is the same as in Kurkela et al. (2014), and has a maximum mass of about $2.5 M_{\odot}$. As seen in the figure, rotating NSs have a larger radius

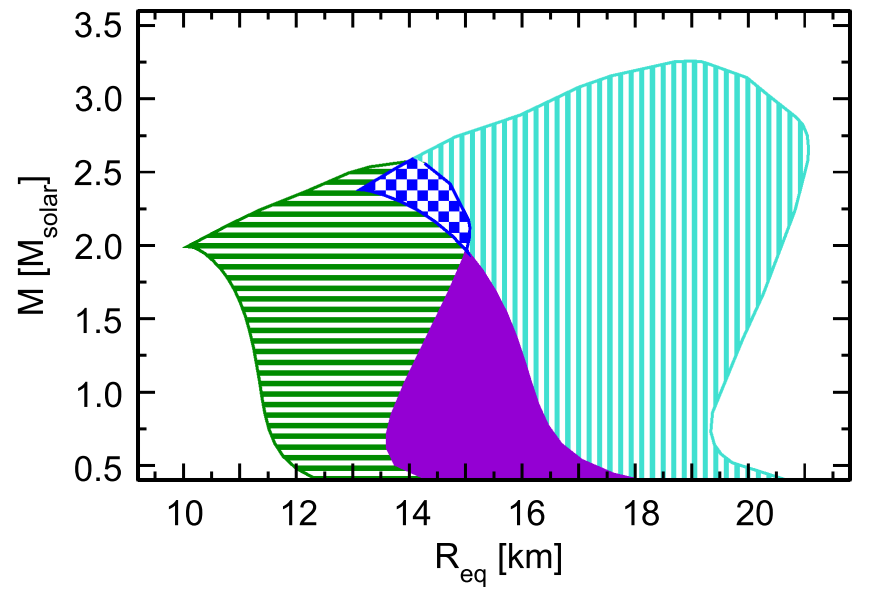

Figure 1. Mass vs. equatorial radius regions for non-rotating stars (horizontal stripes) and mass-shedding stars (vertical stripes). The upper, checkered region is an overlap between the non-rotating and mass-shedding regions. The lower, solid region is only accessible to non-mass-shedding, rotating NSs.

and a larger maximum mass than non-rotating ones. This can be thought of as a consequence of centrifugal force: the stars with large central energy densities that are unstable past the maximum-mass point for non-rotating stars are stabilized (and their central energy densities are lowered) by the outward centrifugal force in the rotating case. The larger radius is a consequence of the eccentricity of the star caused by the centrifugal force as well. We see that the maximum-mass star now has a mass of about $3.25 M_{\odot}$, and the largest star radius is about $21 \mathrm{~km}$.

As one might expect, the boundaries of the non-rotating region and the mass-shedding regions in Figure 1 are formed from the same EoSs; e.g., the EoS that contains the highestmass stars in the non-rotating case also contains the highestmass stars in the mass-shedding case. This means that any further observational constraints that restrict the left, horizontally striped region in Figure 1 will also restrict the right, vertically striped region in the same way.

There is available observational data on the correlated masses and radii of some NSs, in particular, those located in low-mass X-ray binaries (for a recent review, see Ozel \& Freire 2016). Though the uncertainties on these data are sizeable in both mass and radius, considering all of the data together reveals a general region in the mass-radius plane. Moreover, combined analyses can produce still more refined insights. Comparing our allowed mass-radius regions with the results of the analysis of Ozel et al. (2016), we see that our non-rotating region (again, the same as that of Kurkela et al. 2014) fills the larger-radius half of the confidence bands for both the quiescent and thermonuclear-burst data. Moreover, our non-rotating region intersects with the $68 \%$ confidence bands of every NS listed in that work, save one, the quiescent NS labelled "M28," and the confidence band for that star only narrowly misses our region on the side of smaller radii. Finally, the astrophysically inferred mass-radius region presented toward the end of Ozel \& Freire (2016) primarily covers a region of slightly smaller radii than our non-rotating results: the authors" "Astro+Exp" region just touches the smaller-radius edge of our non-rotating results. These observations together seem to favor the EoSs of Kurkela et al. (2014) that produce stars with smaller radii.

In Figure 2, we show the allowed regions for NSs in the mass-frequency plane. The inner, solid region is allowed for 


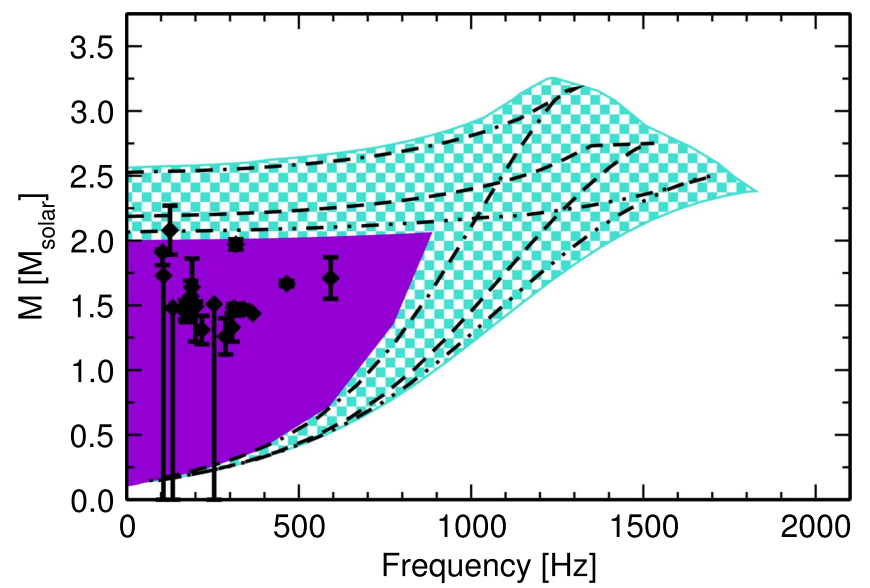

Figure 2. Allowed mass-frequency region for all of the possible EoSs. The inner, solid region is allowed for every equation of state, and the outer, checkered band shows where the possible boundaries are for each EoS. The dashed lines are the outer boundaries of the mass-frequency regions for three sample EoSs. Data points for NSs with $f>100 \mathrm{~Hz}$, taken from a table in Haensel et al. (2016), are also plotted.

every equation of state, and the outer, checkered band shows where the possible boundaries are for each EoS. The right boundary of the checkered region is constrained by the massshedding stars: beyond a certain limiting frequency at a given mass, stars become unstable. The upper boundary of the checkered region consists of the curves $M_{\max }(f)$, with the maximum NS mass as a function of frequency. We also include three dashed lines in Figure 2, which are the boundaries of the mass-frequency regions for three sample EoSs. This is to illustrate the shape of the boundary for each EoS. Every EoS is shaped similarly to these: the top boundary rises toward the sloped, upper-right-hand edge of the checkered region, comes to a point, and then curves back down. Note that this implies that the outermost boundary of the checkered region is not formed from a single EoS; in fact, even the upper edge and right edge of the checkered region are formed by different EoSs.

We also show in Figure 2 data points for NSs with frequencies above $100 \mathrm{~Hz}$, taken from Haensel et al. (2016). A star located in the checkered band would eliminate some of the EoSs (namely, the ones whose curves in the checkered region are closer to the inner, solid region than the data point of the star). We see that there is only one star that is pushing into the checkered band: this is $\mathrm{B} 1516+02 \mathrm{~B}$, with a mass of $2.09 \pm 0.19 M_{\odot}$ (Freire et al. 2008). If the mass of this star were further constrained, it could potentially eliminate a sizeable number of additional EoSs. Note, however, that $f=125.83 \mathrm{~Hz}$ for $\mathrm{B} 1516+02 \mathrm{~B}$, so this is still within the regime where approximating the star as non-rotating is valid. Thus, this constraint is not fundamentally one of rotation.

From Figure 2, however, we see that for high- $f$ stars, there is a constraint coming from rotation. The most clear example of this is the upper-right corner of the inner, solid region with coordinates $(M, f)=\left(2.06 M_{\odot}, 883 \mathrm{~Hz}\right)$. This frequency, $f=883 \mathrm{~Hz}$, signifies the highest frequency that all of the EoSs can support. Thus, if a NS is ever found with $f>883 \mathrm{~Hz}$, this would eliminate some of the possible EoSs of Kurkela et al. (2014) and Fraga et al. (2016). We note, however, that this is the highest frequency that would eliminate some EoSs: lower-frequency NSs could also rule out some EoSs if their

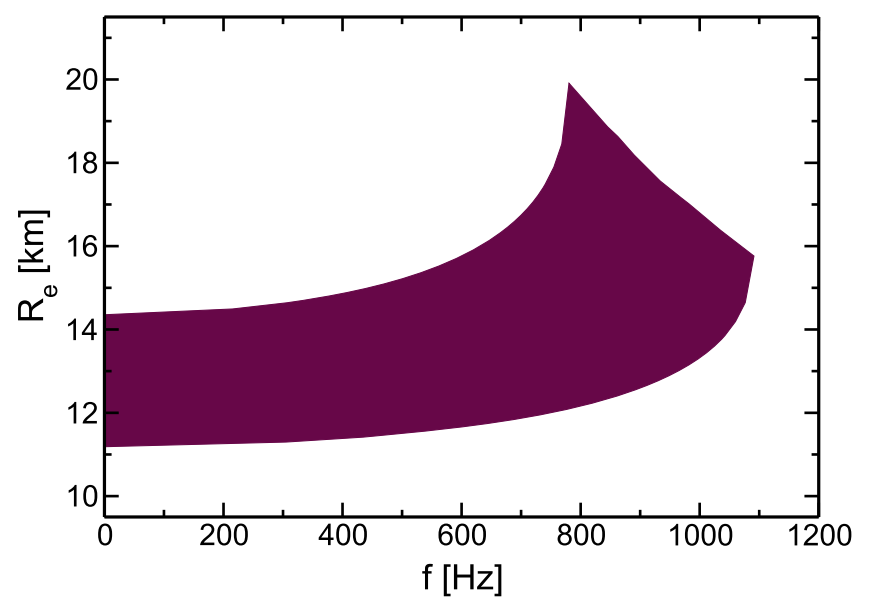

Figure 3. Region of allowed circumferential, equatorial radius vs. frequency curves for a $1.4 M_{\odot}$ star.

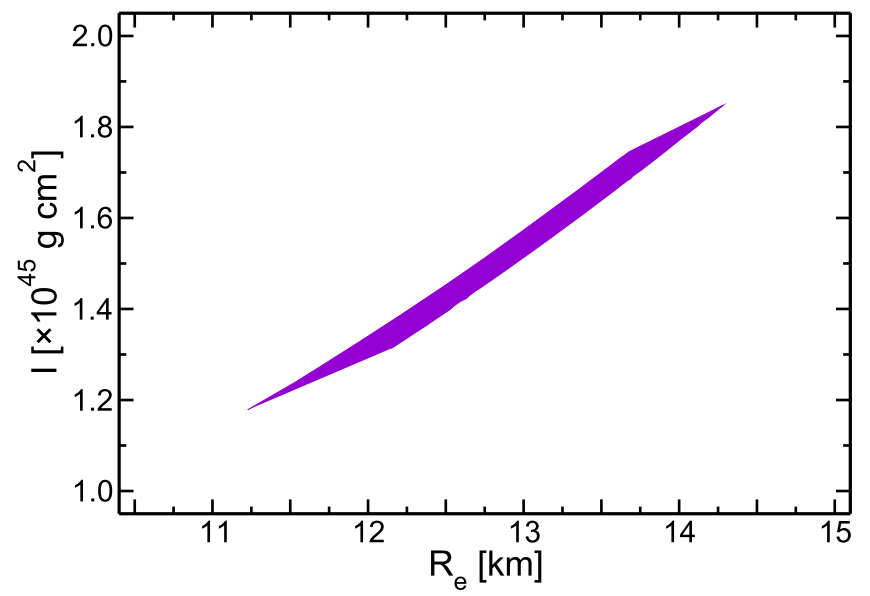

Figure 4. Allowed region of moment of inertia vs. circumferential, equatorial radius for PSR J0737-3039A.

masses could be measured and were sufficiently low. For example, PSR J1748-2446ad, currently the fastest rotating NS known $(f=716 \mathrm{~Hz}$ ) (Hessels et al. 2006), will eliminate some EoSs within this framework if it is found to be less than about $1 M_{\odot}$.

For a $1.4 M_{\odot} \mathrm{NS}$, the largest frequency that all EoSs can support is lower, $f=780 \mathrm{~Hz}$, as show in Figure 3. In this figure, we have plotted the equatorial radius as a function of frequency $R_{\mathrm{e}}(f)$ for a typical $1.4 M_{\odot} \mathrm{NS}$ for each EoS. This plot serves as a prediction for observational astronomers. Furthermore, when consistent, reliable data of NS radii are available, a plot of this type could be overlaid with observational data to further constrain the QCD EoS (similar to Figure 2 above). One other comment we wish to make here is that this radiusfrequency band agrees with the result of the minimum- $\chi^{2}$, hybrid EoS of Kurkela et al. (2010b). That result lies directly in the center of our band in Figure 3. We do note, however, that their mass-frequency boundary only partially agrees with our band: the boundary of the mass-frequency region coming from the mass-shedding curve in Kurkela et al. (2010b) lies in the center of our checkered band coming from our mass-shedding curves, but their upper boundary cuts into our solid band. This is because the minimum- $\chi^{2}$, hybrid EoS obtained in Kurkela et al. (2010b) does not permit a $2 M_{\odot}$ NS. 


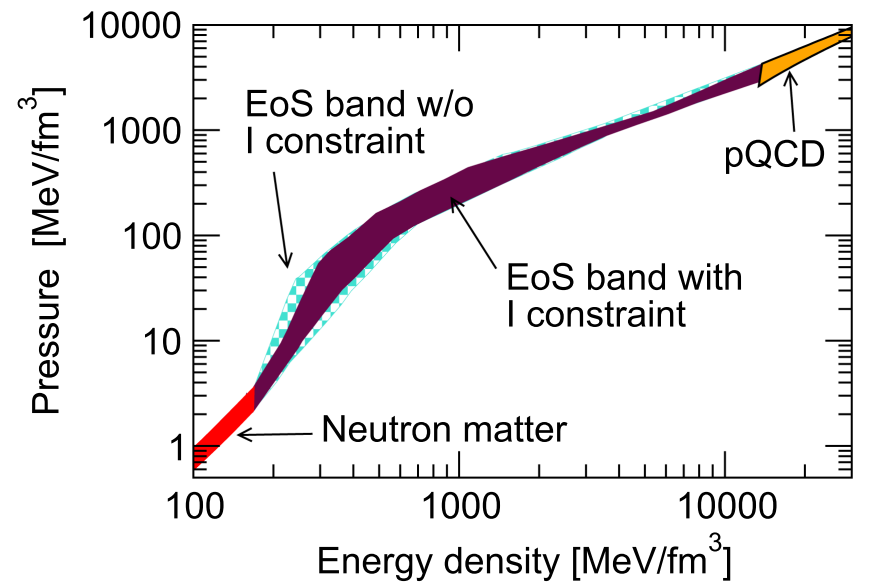

Figure 5. Plot illustrating how much the EoS band from Kurkela et al. (2014) would be restricted by a hypothetical measurement of $I=1.5 \times 10^{45} \mathrm{~g} \mathrm{~cm}^{2}$ with a precision of $10 \%$ for PSR J0737-3039A.

The final plot that we have generated from the EoSs is shown in Figure 4. In this figure, we show the allowed region for the moment of inertia and equatorial radius of PSR J0737-3039A. The moment of inertia of this star may be measured in a few years (Morrison et al. 2004; Kramer \& Wex 2009), and so it is natural to investigate what the QCD EoSs predicts its value should be. We find that $I \in[1.2,1.8] \times 10^{45} \mathrm{~g} \mathrm{~cm}^{2}$. Work of this type has been performed previously assuming phenomenological EoSs, e.g., in Lattimer \& Schutz (2005), Morrison et al. (2004), and Bejger et al. (2005), and, more recently, Raithel et al. (2016) have performed an analysis in which an EoS is only assumed up to $n_{s}$, and the remaining mass is shifted around to minimize and maximize $I$ for the star. This allows the authors to plot the largest allowed region in the $R_{e}, I$ plane constrained by controlled, first-principles, low-energy physics. Our allowed region in Figure 4 does fall within the larger- $R_{\mathrm{e}}$, larger- $I$ (i.e., upper-right) portion of the region calculated in the aforementioned work, and it also falls roughly in the center of the 40 EoSs data points presented in an earlier figure in that work.

We also find that all of the "hard" and "soft" EoSs from Kurkela et al. (2014) and Fraga et al. (2016) fall on the two boundaries of our allowed region: the "hard" EoSs form the right boundary and the "soft" ones form the left boundary. In other words, the "hard" and "soft" EoSs each lie on their own fixed curve. This is not surprising, since the largest contribution to $I$ comes from the matter at the largest radii (in the lowdensity crust region), and there, all the "hard" or "soft" EoSs agree by construction. However, since these EoSs form the vertical boundaries of the region, even a relatively imprecise measurement of the moment of inertia of PSR J0737-3039A (e.g., one with a precision of $10 \%$ ) will significantly constrain which EoSs are consistent with the measurement. Since the allowed region spans $0.6 \times 10^{45} \mathrm{~g} \mathrm{~cm}^{2}$ in $I$, a $10 \%$ measurement will only be consistent with about $0.15 / 0.6=25 \%$ of the EoSs.

This percentage is not a physical meaningful result, but we translate it into a statement about the equation of state band in Figure 5. In this figure, we display the Kurkela et al. EoS band, along with the subset of it that is consistent with $I=1.5 \times 10^{45} \mathrm{~g} \mathrm{~cm}^{2}$ to a precision of $10 \%$, as an example. We see that such a measurement would shrink the percent errors of the band by up to $50 \%$ in some places, especially in the lowest-density regime. Again, this makes sense because it is the low-density material farthest from the rotation axis that contributes most to $I$. This reduction in the EoS band would then, by extension, significantly constrain all of the NS properties mentioned in this work. This makes a measurement of $I$ of the double pulsar PSR J0737-3039A of extreme interest. Such a measurement would also constrain the radius of the pulsar to within about $\pm 0.5 \mathrm{~km}$ in the context of the EoSs used in this work.

\section{CONCLUSIONS}

In this work, we have investigated the effects of rotation on global properties of NSs constructed from the EoSs of Kurkela et al. (2014) and Fraga et al. (2016). We have found the maximum allowed NS mass to be about $3.25 M_{\odot}$ and the maximum allowed NS radius to be about $21 \mathrm{~km}$. From investigations of mass-radius relations, we have observed that the smaller-radius results of Kurkela et al. (2014) are favored by current data and analyses. From investigations of massfrequency relations, we have identified B1516+02B as an NS of particular interest: constraining its mass more precisely could potentially eliminate many allowed QCD EoSs. From mass-frequency relations, we have also identified $f=883 \mathrm{~Hz}$ as the maximum allowed NS rotation frequency consistent with every EoS. In the case of a canonical $1.4 M_{\odot} \mathrm{NS}$, we have found that $f=780 \mathrm{~Hz}$ is the maximum allowed rotation frequency consistent with every EoS. We have also determined the allowed $R_{\mathrm{e}}$ versus $f$ region for a $1.4 M_{\odot}$ NS, which may serve has a prediction for astronomers, and may also be overlaid with future precise radius measurements to further constrain the QCD EoS. Finally, we have calculated the moment of inertia and radius of PSR J0737-3039A for each EoS and found it to be consistent with the minimally constrained results of Raithel et al. (2016). We have found that $I \in[1.2,1.8] \times 10^{45} \mathrm{~g} \mathrm{~cm}^{2}$ for the allowed QCD EoSs. Most excitingly, we have concluded that even a measurement of the moment of inertia of this star with a precision of $10 \%$ would reduce the percent errors of the band of allowed QCD EoSs consistent with observations to $50 \%$ of its current size at low densities. We thus conclude that a measurement of the moment of inertia of PSR J0737-3039A would be of extreme interest.

The author would like to thank Aleksi Kurkela, Paul Romatschke, and Aleksi Vuorinen for many helpful discussions and suggestions.

\section{REFERENCES}

Abbar, S., Carlson, J., Duan, H., \& Reddy, S. 2015, PhRv, C92, 045809 Antoniadis, J., Freire, P., Wex, N., et al. 2013, Sci, 340, 6131 Bejger, M., Bulik, T., \& Haensel, P. 2005, MNRAS, 364, 635

Benhar, O., Ferrari, V., Gualtieri, L., \& Marassi, S. 2005, PhRv, D72, 044028 Cipolletta, F., Cherubini, C., Filippi, S., Rueda, J. A., \& Ruffini, R. 2015, PhRv, D92, 023007

Demorest, P., Pennucci, T., Ransom, S., Roberts, M., \& Hessels, J. 2010, Natur, 467, 1081

Fraga, E. S., Kurkela, A., \& Vuorinen, A. 2016, Eur. Phys. J., A52, 49

Freire, P. C. C., Wolszczan, A., Berg, M. v. d., \& Hessels, J. W. T. 2008, ApJ, 679,1433

Glendenning, N. 2000, Compact Stars: Nuclear Physics, Particle Physics, and General Relativity (2nd ed.; New York: Springer)

Glendenning, N. K. 1992, PhRv, D46, 1274

Haensel, P., Bejger, M., Fortin, M., \& Zdunik, L. 2016, Eur. Phys. J., A52, 59

Hebeler, K., \& Schwenk, A. 2010, PhRv, C82, 014314 
Hessels, J. W. T., Ransom, S. M., Stairs, I. H., et al. 2006, Sci, 311, 1901 Kramer, M., \& Wex, N. 2009, CQGra, 26, 073001

Kurkela, A., Fraga, E. S., Schaffner-Bielich, J., \& Vuorinen, A. 2014, ApJ, 789, 127

Kurkela, A., Romatschke, P., \& Vuorinen, A. 2010a, PhRv, D81, 105021

Kurkela, A., Romatschke, P., Vuorinen, A., \& Wu, B. 2010b, arXiv:1006.4062

Lattimer, J. M., \& Schutz, B. F. 2005, ApJ, 629, 979
Morrison, I. A., Baumgarte, T. W., Shapiro, S. L., \& Pandharipande, V. R. 2004, ApJL, 617, L135

Ozel, F., \& Freire, P. 2016, ARA\&A, 54, 401

Ozel, F., Psaltis, D., Guver, T., et al. 2016, ApJ, 820, 28

Raithel, C. A., Ozel, F., \& Psaltis, D. 2016, PhRv, C93, 032801 [Addendum: PhRvC93, no.4,049905(2016)]

Tews, I., Krüger, T., Hebeler, K., \& Schwenk, A. 2013, PhRvL, 110, 032504 